\title{
BMJ Open General practitioners' awareness of the recommendations for faecal immunochemical tests (FITs) for suspected lower gastrointestinal cancers: a national survey
}

\author{
Christian Von Wagner, ${ }^{1}$ Sandro Tiziano Stoffel, ${ }^{1}$ Madeline Freeman, ${ }^{1}$ \\ Helga E Laszlo, ${ }^{2}$ Brian D Nicholson, ${ }^{3}$ Jessica Sheringham, ${ }^{4}$ Dorothy Szinay, ${ }^{1}$ \\ Yasemin Hirst ${ }^{1}$
}

To cite: Von Wagner C, Stoffel ST, Freeman M, et al. General practitioners' awareness of the recommendations for faecal immunochemical tests (FITs) for suspected lower gastrointestinal cancers: a national survey. BMJ Open 2019;9:e025737. doi:10.1136/ bmjopen-2018-025737

- Prepublication history and additional material for this paper are available online. To view these files, please visit the journal online (http://dx.doi. org/10.1136/bmjopen-2018025737).

Received 2 August 2018 Revised 23 January 2019 Accepted 5 March 2019

\section{Check for updates}

(C) Author(s) (or their employer(s)) 2019. Re-use permitted under CC BY-NC. No commercial re-use. See rights and permissions. Published by BMJ.

${ }^{1}$ Research Department of Behavioural Science and Health, UCL, London, UK

${ }^{2}$ UCLH Cancer Collaborative, UCLH, London, UK

${ }^{3}$ Nuffield Department of Primary Care Health Sciences, University of Oxford, Oxford, UK

${ }^{4}$ Department of Applied Health Research, University College London, London, UK

Correspondence to Dr Christian Von Wagner; c.wagner@ucl.ac.uk

\section{ABSTRACT}

Objectives In July 2017, UK National Institute for Health and Care Excellence (NICE) published a diagnostic guidance (DG30) recommending the use of faecal immunochemical tests (FITs) for symptomatic patients who do not meet the urgent referral pathway for suspected colorectal cancer (CRC). We assessed general practitioners' (GP) awareness of DG30 in primary care 6 months after its publication.

Design and setting Cross-sectional online survey of GPs hosted by an English panel of Primary health care professionals.

Participants In December 2017, 1024 GPs registered on an online panel (M3) based in England took part in an online survey.

Outcomes and variables We investigated a number of factors including previous experience of using FIT and guaiac faecal occult blood tests (FOBTs), the number of urgent referrals for CRC that GPs have made in the last year and their sociodemographic and professional characteristics that could be associated with their self-reported awareness of the FIT diagnostic guidance.

Results Of the $1024 \mathrm{GPs}$ who completed the survey, $432(42.2 \%)$ were aware of the current recommendation but only $102(10 \%)$ had used it to guide their referrals. Awareness was lowest in North West England compared with London (30.5\% vs $44.9 \%$; adjusted OR: 0.55 , $95 \% \mathrm{Cl} 0.33$ to 0.92$)$. Awareness of the FIT guidance was positively associated with test usage after the NICE update (adjusted OR: $13.00,95 \% \mathrm{Cl} 6.87$ to 24.61) and having specialist training (adjusted OR: 1.48, $95 \% \mathrm{Cl} 1.05$ to 2.08$)$. The number of urgent referrals, the previous use of FOBt, GPs' age and gender, work experience and practice size (both in terms of the number of GPs or patients at the practice) were not associated with awareness.

Conclusions Less than half of GPs in this survey recognised the current guidance on the use of FIT. Selfreported awareness was not systematically related to demographic of professional characteristics.

\section{Strengths and limitations of this study}

- This is the first study to date to report on general practitioner (GP) awareness of the new diagnostic guidance (DG30) on the use of home-based stool testing.

- The survey benefited from a large national online panel of GPs.

- Only a small proportion of GPs responded to the survey potentially undermining the representativeness of our findings.

\section{INTRODUCTION}

Although organised colorectal cancer (CRC) screening offers the best chance for early detection at an asymptomatic stage, it only accounts for $10 \%$ of all CRC diagnosis. A third of the patients who are symptomatic are diagnosed through the urgent cancer referral route (2-week wait $[2 \mathrm{WW}]$ ) and a quarter are diagnosed following an emergency presentation. ${ }^{1}$

Currently, the pathways for symptomatic patients are outlined in the National Institute for Health and Care Excellence's (NICE) Suspected Cancer: Recognition and Referral Guideline (NG12). ${ }^{2}$ In 2015, the CRC guidelines were updated to include vague symptoms such as weight loss and abdominal pain, which aimed to increase the number of cancers diagnosed by the fast-tracked 2WW-pathway.

The 2015 guideline has also recommended the use of guaiac-based faecal occult blood tests (FOBts) to safety-net patients whose symptoms do not warrant an immediate colonoscopy investigation. However, clinicians have previously criticised FOBt for its low accuracy and general practitioners (GPs) 
were discouraged to use this test to guide their referral decisions. ${ }^{3}$

More recently, there has been increasing evidence for promoting the use of quantitative faecal immunochemical tests (FITs) in primary care as a point of care test to triage patients that present with symptoms but do not meet the $2 \mathrm{WW}$ referral criteria. ${ }^{45}$ The primary advantage of FIT for symptomatic patients is its potential to reduce the number of unnecessary colonoscopies, thus avoiding associated risks from the procedure and free up endoscopy capacity that can be reused to carry out procedures for high-risk patients. ${ }^{6-8}$ There is a growing body of evidence that the FIT test with a diagnostic threshold of $10 \mu \mathrm{g}$ of haemoglobin per gram of faeces could be used to guide GP referral for further investigation for significant bowel diseases. ${ }^{9-12}$ Thus, in July 2017, the NICE has published a diagnostic guidance (DG30) and subsequently updated NG12 to replace the FOBt with a more accurate FIT test. ${ }^{13}$

All NICE guidelines are freely available online. Their adoption of primary care is a key challenge to implementation as they are not mandatory unlike organised cancer screening programmes that are implemented nationally. A recent Delphi survey on the adoption of NICE recommendations in primary care suggests that while GPs agree with the importance and validity of evidence-based recommendations, their adoption will depend on the brevity of the guidelines, the communication strategies to make it accessible and the relevance of the evidence to primary care patients. ${ }^{14}$ Furthermore, their adoption depends on how GPs have been informed about the recommendations by the local or national authorities. ${ }^{15} 16$ Another important consideration is whether recommendations are being positioned as a guidance or as a guideline. According to the NICE website, ${ }^{17}$ a guideline provides recommendations on how care and services should be provided for a specific condition ranging from provision of clinical guidelines to safe staffing guidelines. Conversely, a specific guidance is less binding but aims to give evidence-based support to healthcare professionals making decisions on the use of specific innovations. NICE guidance (NG) includes recommendations on technology, prescriptions as well as diagnostic technologies such as FIT.

Only a handful of studies have investigated the barriers and facilitators of the implementation of specific NICE recommendations, eg, adoption of the NG12 guideline and the clinical guideline for breast cancer (CG164) ${ }^{18-20}$ Among those studies, lack of awareness (eg, only $24 \%$ of GPs participating in an online study were aware of the CG164 recommending the use of tamoxifen to women with increased risk of breast cancer) was cited as a major barrier for GPs to prescribe the drug. ${ }^{19}$ Low awareness could explain why a subsequent prospective study using health records showed low adherence to the recommendations for prescribing tamoxifen since 2013. ${ }^{20}$

Furthermore, a recent study from Denmark reported that GPs' capability of using FIT was limited by their awareness of the test kit and perceived lack of support from other GPs and practice staff. ${ }^{21}$ The decision to commission the use of FIT in symptomatic primary care patients is made by local Clinical Commissioning Groups (CCGs). While they are national FIT guidelines, each CCG has the autonomy to respond in the most appropriate way to meet local requirements. At the time of the survey, there were clear examples of how CCGs had responded to the NG12 FIT guidance in the adoption resource published with the DG30 guidance. ${ }^{13}$ For example, in Oxfordshire a series of outreach educational sessions were delivered by the CCG accompanied by email educational bulletins to all GPs and information posted on the Oxford University Hospitals laboratory webpages.

Thus, the present study aimed to assess GPs' self-reported awareness of the FIT recommendation across England for patients who do not meet the 2WW criteria since DG30 was published in July 2017.

\section{METHODS}

\section{Study design and population}

The data were collected as part of a 10 min online survey in December 2017 that aimed to investigate GPs' acceptability of FIT in primary care as a rule-out test of CRC among symptomatic patients. ${ }^{22} 14100 \mathrm{GPs}$ from England were invited to take part via email using M3 Global Research's panel of 41935 GPs and health professionals in England.

GPs were eligible if they were working in England at the time of the study. Once they agreed to take part, they were provided with information on the current NICE guidelines and the DG30 update as part of the information provided on FIT as a rule-out test (see online supplementary materials).

Duplicates and incomplete data were excluded from the final analysis. The survey was hosted by an online cloud-based survey administration company called Survey Monkey Limited and the order of the questions was randomised. Only researchers at University College London (UCL) had access to the data. GPs were offered an industry standard honorarium.

\section{Patient and public involvement}

This work forms part of the University College London Hospita (UCLH) Cancer Collaborative whose overall strategy is informed through consultation with patient representatives. As the main topic of this survey was directed at healthcare professionals and their decision making its development was informed by previous interviews and consultation with GPs, particularly with one of our coauthors (BDN).

\section{Measures}

Self-reported awareness of the diagnostic guidance recommendation on using FIT (DG30)

After having been presented with information about current recommendation, respondents were asked 
whether they recognised guidance on the use of FIT as a triage test for low-risk patients who do not meet the $2 \mathrm{WW}$ criteria based on DG30

("Before today, were you aware that FIT can be used in primary care to rule in [detect] colorectal cancer among patients with colorectal cancer symptoms among low risk patients?").

\section{GPs' demographic and professional characteristics}

GPs were asked to provide their age range, gender, years of experience as an active GP and role in their practice. GPs were also asked to provide details of the region in England where they are based.

A recent study on the implementation of NICE recommendations in healthcare settings suggests involvement in budget setting and clinical engagement are important facilitators of adoption of new guidelines. ${ }^{23}$ Thus, we additionally asked GPs about their engagement in implementation/budget planning, role as a cancer lead, engagement in research, the number of registered patients and the number of GPs working in their practice.

GPs were also asked to indicate if they had received specialist training. A summary of respondents' specialisms is listed in the online supplementary materials. For the purpose of the analysis, we dichotomised respondents as those who did or did not receive specialist training.

\section{Previous experience using faecal tests}

Previous experience of requesting FOBt and FIT for symptomatic patients based on the NICE Guideline (NG12) or recent diagnostic guidance (DG30) was assessed using dichotomous (Yes/No) items.

Number of 2WW referrals for suspected CRC in the last 12 months We asked GPs to indicate the number of $2 \mathrm{WW}$ referrals they had made over the past 12 months.

\section{Statistical analysis}

Simple logistic regression analyses were used to look at bivariate and multivariate associations between awareness of diagnostic guidance (DG30) and GPs demographic and professional characteristics, previous use of faecal tests for symptomatic patients and also the number of $2 \mathrm{WW}$ referrals they had personally made in the last year. The results were reported using percentages, unadjusted ORs, adjusted ORs (aORs) and 95\% CIs. All analyses were completed using SPSS V.23.

\section{RESULTS}

\section{Population characteristics}

The sample characteristics have been reported previously (22). In brief, just under $10 \%$ of the GPs responded to the invitation to the online survey $(n=1351)$. Of the 1351 GPs, $24.2 \%$ of the initial respondents either dropped out of the survey during data collection $(n=209)$ or were excluded because they did not qualify or had already completed survey previously $(\mathrm{n}=118)$. In total, 1024 GPs from England successfully completed the survey.
Self-reported awareness of FIT diagnostic guidance for symptomatic low-risk patients

$42.2 \%(n=432)$ of respondents were aware of the use of FIT as a triage test for low-risk patients based on DG30. About half of the respondents $(54.4 \%, \mathrm{n}=558)$ stated that they used FOBt for low-risk patients according to NG12 published in 2015 and only about $10 \%(\mathrm{n}=102)$ used FIT since it was updated in July 2017 following the publication of DG30.

Table 1 shows the unadjusted and adjusted regression analysis. Respondents were significantly more likely to be aware of the FIT recommendation if they had requested low-risk patients to complete FIT since July 2017 compared with those who had not $(88.2 \%$ vs $37.1 \%$; aOR $13.00,95 \%$ CI 6.87 to 24.61). GPs with any specialist training were more likely to be aware of the FIT recommendation for low-risk patients compared with those who had not received specialist training $(50.5 \%$ vs $39.9 \%$; aOR $1.48,95 \%$ CI 1.05 to 2.08). Respondents were less likely to be aware of the FIT recommendation if they were working in North West England compared with London (30.5\% vs $44.9 \%$; aOR $0.55,95 \%$ CI 0.33 to 0.92 ). There were no differences in respondents' awareness of the FIT recommendation by GPs age, gender and employment status, number of years working as a GP and having a role in budget setting.

\section{DISCUSSION}

This survey provides insights about GPs' awareness of the latest FIT recommendations in the National Guideline for Suspected Cancer: Recognition and Referral. Even though $42 \%$ of participants being aware of DG 30 was higher than what has been cited for other clinical guidelines (eg, CG164) ${ }^{19}$ our findings suggest that awareness of the diagnostic guidance approximately 6 months after their publication is still relatively low and geographically varied. In particular, GPs who took part in this study were less likely to be aware of the update, if they were based in the North West of England.

As it stands, the evidence on GP awareness and the adoption of NICE guidelines for suspected CRC is scarce. The International Cancer Benchmarking Partnership study on adherence to national guidelines suggest that less than half of GPs in the UK adhere to the CRC referral guidelines and the majority will ignore the guidelines if they see a patient with symptoms suggestive of CRC. ${ }^{18}$ This was further supported in recent papers that GPs are currently more inclined to use $2 \mathrm{WW}$ referrals to rule out CRC, and they perceive safety netting in cancer diagnosis (eg, watchful waiting and telling patients to come back if the symptoms persist) to be ineffective and potentially leading to diagnostic delays. ${ }^{6}{ }^{23}$ It is not clear to what extent FIT can address these concerns particularly as FIT bears a close family resemblance to the FOBt which is used in the asymptomatic population and often viewed critically by the primary care community due to its low sensitivity. ${ }^{323}$

Furthermore, the outcomes of this study provide the baseline and an early indication for the adoption of the 
Table 1 Unadjusted and adjusted logistic regression models

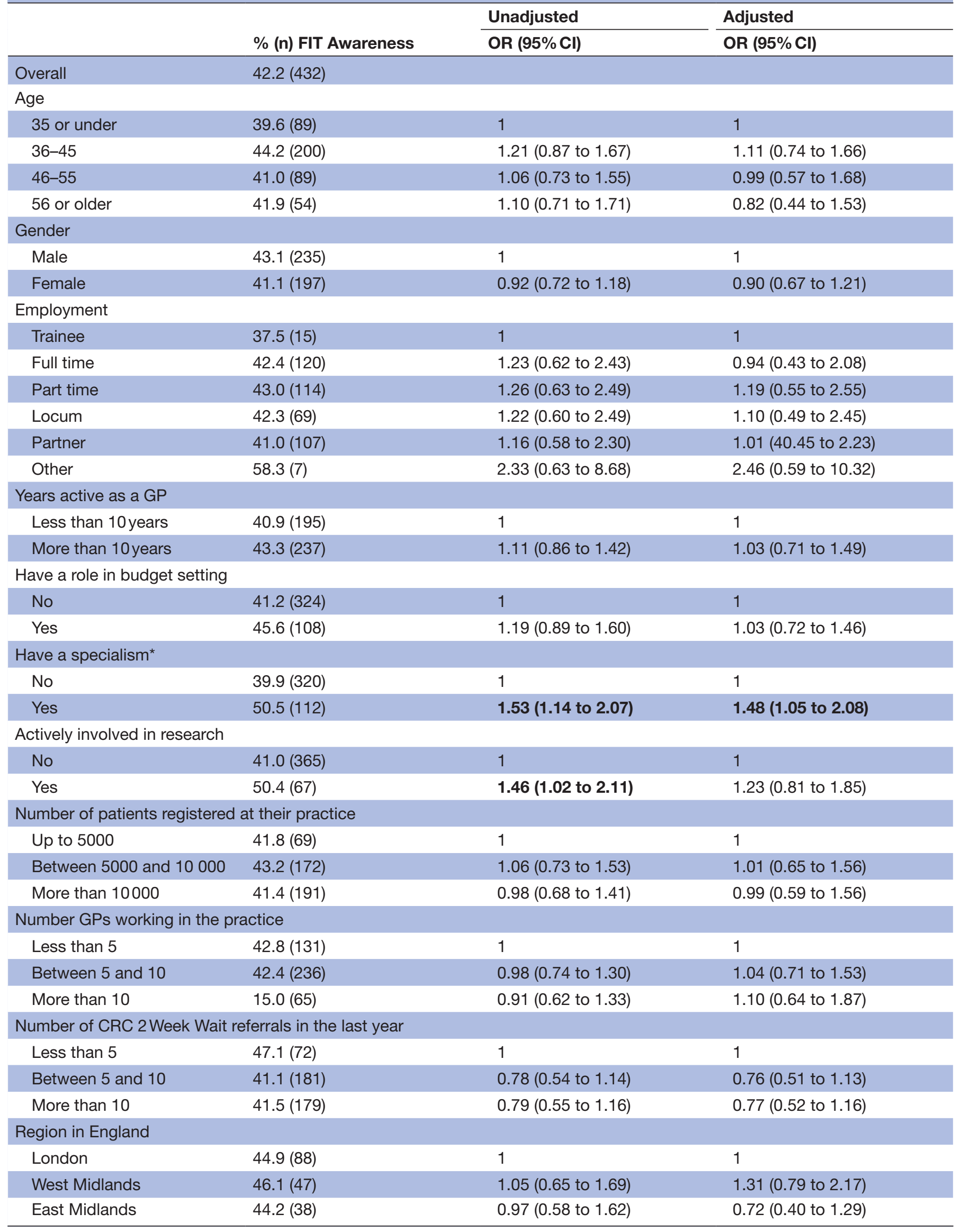




\begin{tabular}{|c|c|c|c|}
\hline & & Unadjusted & Adjusted \\
\hline & $\%$ (n) FIT Awareness & OR (95\% Cl) & OR $(95 \% \mathrm{Cl})$ \\
\hline South West & $37.8(42)$ & $0.75(0.46$ to 1.20$)$ & $0.90(0.54$ to 1.49$)$ \\
\hline East of England & $47.7(62)$ & $1.12(0.80$ to 1.87$)$ & $1.26(0.78$ to 2.04$)$ \\
\hline $\begin{array}{l}\text { Yorkshire and the Humber } \\
\text { and North East }\end{array}$ & $33.3(38)$ & 0.61 (0.38 to 0.99$)$ & $0.75(0.45$ to 1.24$)$ \\
\hline \multicolumn{4}{|c|}{ Previous experience using FOBt } \\
\hline No & $39.9(186)$ & 1 & 1 \\
\hline Yes & $44.1(246)$ & 1.19 (0.93 to 1.52$)$ & $0.96(0.73$ to 1.28$)$ \\
\hline \multicolumn{4}{|l|}{ Previous experience using FIT } \\
\hline
\end{tabular}

The results highlighted in bold indicate $p$ values less than 0.05 .

$\mathrm{CRC}$, colorectal cancer; FIT, faecal immunochemical test; GP, general practitioner.

new diagnostic guidance on FIT. From it, there is no clear profile emerging as early adopters other than, geographical location and having received additional specialist training.

One explanation of GPs' low awareness of the guidance may relate to the existing low access to faecal occult blood tests $(\sim 54 \%)$ compared with other direct laboratory tests provided in primary care such as Ca125 which had 100\% access across England. ${ }^{24}$

Furthermore, a recent study on the implementation of evidence-based guidelines suggests that current implementation strategies adopt a top-down approach which often lacks sufficient clarity and fails to address local circumstances. ${ }^{25}$ This may also particularly apply to DG30 because most evidence was collected through secondary care and informed by patients who had already been referred to $2 \mathrm{WW}$ referrals investigations and evidence did not to consider the use of FIT as a stand-alone test without the added reassurance of a colonoscopy. ${ }^{4}$

Currently, the small number of local FIT initiatives since July 2017 suggest that there might be important variation in access to FIT in primary care. ${ }^{26}$ Low awareness of GPs is therefore likely to reflect macro-level variation in local commissioning authorities acting on the new diagnostic guidance on how to use FIT (DG30). However, it was noteworthy that even in areas where FIT has been implemented (eg, Oxfordshire, South-West England) awareness remained suboptimal (38\%).

According to the NICE adoption support guidance published in January $2018,{ }^{26}$ key stakeholders including CCGss, clinicians, laboratories, GPs and local trusts should ensure that there is sufficient local preparation. Most important here would be to develop standard operating procedures detailing safety-netting procedures for patients who do not return test kits. In addition, it is important to develop pathways to follow-up FIT results with the appropriate safety netting to ensure patients with normal and abnormal follow-up clinical advice.

Importantly, local guideline development should involve active participation from local GPs and best practice examples using local pilot sites to enhance engagement with the guidelines. ${ }^{15}$ Given that awareness is currently low, there is a need to monitor progress in improved recognition and adoption. Local commissioners should invest in primary care education and training activities as part of the implementation activities. Such activities would be particularly important in areas with the lowest awareness (eg, North West England).

The primary strength of this study was the use of a very large sample of GPs practising in England whose characteristics were very similar to those reported in a previous GP-based online survey. ${ }^{18}$ The primary outcome question was embedded in a wider survey on GP acceptability of FIT as an alternative to a $2 \mathrm{WW}$ referral for patients with symptoms suggestive of CRC. As a result, we were limited in the scope of questions we could ask about recognition of the new diagnostic guidance (DG30) and its implementation in primary. Future surveys would gain deeper insights by addressing additional aspects such as GPs' intended and actual use FIT for low-risk patients, the sources where GPs gather and learn new information on NG and factors influencing their ability to follow guidelines and guidance (eg, implementation by local authorities). As this study was cross-sectional, we do not know what GPs baseline knowledge of FIT and its role in diagnostics was before the introduction of the guideline. Future studies using longitudinal data would also enable us to disentangle the nature of the relationship between awareness and actual FIT use in practice (eg, whether awareness prompts FIT use or whether seeking out to use 
FIT prompts GPs to review clinical referral guidance and guidelines). Especially given the low response rate means that we cannot exclude the possibility that awareness might be lower. Relatedly, we acknowledge that while we feel that ORs made our results easier to interpret, their values might be inflated compared with risk ratios.

\section{CONCLUSION}

Our survey findings suggest that GPs' awareness of using FIT as a triage test in primary care is currently low and there is limited insight into the perceived barriers and facilitators associated with GPs' use of the test. Successful early adoption of the FIT test in primary care will require extensive primary care engagement to raise awareness of the NICE guideline and the updated diagnostics guidance in parallel with providing access to the FIT test.

Acknowledgements We thank Professor Georgios Lyratzopoulos for his support and advise throughout the project. We also thank Professor Kathy Pritchard Jones and Mairead Lyons for their guidance with the study and the manuscript.

Contributors CVW, YH and HEL conceived the study. CVW, YH, HEL, MF, BDN, JS, STS and DS contributed to the questionnaire design. STS, YH and CVW monitored data collection. STS and YH conducted the data analysis. YH, CVW and STS drafted the manuscript. CVW, YH, HEL, MF, BDN, JS, STS and DS commented and contributed to the further drafts.

Funding This project, as part of the qFIT pilot research study (IRAS 213710), was funded by the NHS England Cancer Vanguard Programme and delivered by the UCLH Cancer Collaborative. YH, STS and MF are funded by a Cancer Research UK Programme grant awarded to Prof Jane Wardle (C1418/A14134). BDN is funded by Macmillan as the GP Facilitator for Oxfordshire. JS was supported by theNational Institute for Health Research (NIHR) Collaboration for Leadership in Applied Health Research and Care North Thames at Barts Health NHS Trust. The views expressed are those of the author(s) and not necessarily those of the NHS, the NIHR or the Department of Health and Social Care.

Competing interests None declared.

Patient consent for publication Not required.

Ethics approval Ethical approval for the study was awarded by University College London Research Ethics Committee (11381/001).

Provenance and peer review Not commissioned; externally peer reviewed.

Data sharing statement Data will be made available to other researchers after the acceptance for publication of the main findings of the qFIT pilot: GP acceptability survey. Data requests will be assessed on a case-by-case basis. Users will be required to complete a data sharing agreement.

Open access This is an open access article distributed in accordance with the Creative Commons Attribution Non Commercial (CC BY-NC 4.0) license, which permits others to distribute, remix, adapt, build upon this work non-commercially, and license their derivative works on different terms, provided the original work is properly cited, appropriate credit is given, any changes made indicated, and the use is non-commercial. See: http://creativecommons.org/licenses/by-nc/4.0/.

\section{REFERENCES}

1. Health and Social Care Information Centre. National bowel cancer audit report 2015. 2015 https://files.digital.nhs.uk/publicationimport/ pub19xxx/pub19500/nati-clin-audi-bowe-canc-2015.pdf (accessed 30 Oct 2018).

2. National Institute for Health and Care Excellence. Suspected cancer: Recognition and referral. NICE guideline (NG12, 2017. [updated 2017]. https://www.nice.org.uk/guidance/ng12. (Accessed 30 Oct 2018).

3. Steele R, Forgacs I, McCreanor G, et al. Use of faecal occult blood tests in symptomatic patients. BMJ 2015;351:h4256.

4. Westwood M, Lang S, Armstrong N, et al. Faecal immunochemical tests (FIT) can help to rule out colorectal cancer in patients presenting in primary care with lower abdominal symptoms: a systematic review conducted to inform new NICE DG30 diagnostic guidance. BMC Med 2017;15:189.

5. Westwood M, Corro Ramos I, Lang S, et al. Faecal immunochemical tests to triage patients with lower abdominal symptoms for suspected colorectal cancer referrals in primary care: a systematic review and cost-effectiveness analysis. Health Technol Assess 2017;21:1-234.

6. Forgacs I, Ashton R, Allum W, et al. Conference report: improving outcomes for gastrointestinal cancer in the UK. Frontline Gastroenterol 2018;9:49-61.

7. Digby J, Fraser CG, Carey FA, et al. Interval cancers using a quantitative faecal immunochemical test (FIT) for haemoglobin when colonoscopy capacity is limited. J Med Screen 2016;23:130-4.

8. Lawler M, Alsina D, Adams RA, et al. Critical research gaps and recommendations to inform research prioritisation for more effective prevention and improved outcomes in colorectal cancer. Gut 2018;67:179-93.

9. Mowat C, Digby J, Strachan JA, et al. Faecal haemoglobin and faecal calprotectin as indicators of bowel disease in patients presenting to primary care with bowel symptoms. Gut 2016;65:1463-9.

10. McDonald PJ, Digby J, Innes C, et al. Low faecal haemoglobin concentration potentially rules out significant colorectal disease. Colorectal Dis 2013;15:e151-e159.

11. Godber IM, Todd LM, Fraser CG, et al. Use of a faecal immunochemical test for haemoglobin can aid in the investigation of patients with lower abdominal symptoms. Clin Chem Lab Med 2016;54:595-602.

12. Cubiella J, Salve M, Díaz-Ondina M, et al. Diagnostic accuracy of the faecal immunochemical test for colorectal cancer in symptomatic patients: comparison with NICE and SIGN referral criteria. Colorectal Dis 2014;16:0273-O282.

13. National Institute for Health and Care Excellence. Quantitative faecal immunochemical tests to guide referral for colorectal cancer in primary car. Diagnostics guidance (DG30). 2017. https://www.nice. org.uk/guidance/dg30 (Accessed 30 Oct 2018).

14. Abdelhamid A, Howe A, Stokes $T$, et al. Primary care evidence in clinical guidelines: a mixed methods study of practitioners' views. $\mathrm{Br}$ $J$ Gen Pract 2014;64:e719-e727.

15. Lowson $\mathrm{K}$, Jenks M, Filby A, et al. Examining the implementation of NICE guidance: cross-sectional survey of the use of NICE interventional procedures guidance by NHS Trusts. Implement Sci 2015;10:93.

16. Atkins L, Kelly MP, Littleford C, et al. Reversing the pipeline? Implementing public health evidence-based guidance in english local government. Implement Sci 2017;12:63.

17. National Institute for Health and Care Excellence. NICE Guidance. 2018. https://www.nice.org.uk/about/what-we-do/our-programmes/ nice-guidance (Accessed 30 Oct 2018).

18. Nicholson BD, Mant D, Neal RD, et al. International variation in adherence to referral guidelines for suspected cancer: a secondary analysis of survey data. Br J Gen Pract 2016;66:e106-e113.

19. Smith SG, Foy R, McGowan JA, et al. Prescribing tamoxifen in primary care for the prevention of breast cancer: a national online survey of GPs' attitudes. Br J Gen Pract 2017;67:e414-e427.

20. Curtis HJ, Walker AJ, Goldacre B. Impact of NICE guidance on tamoxifen prescribing in England 2011-2017: an interrupted time series analysis. Br J Cancer 2018;118:1268-75.

21. Juul JS, Vedsted P, Bro F. Development of an intervention for implementing immunochemical faecal occult blood test in general practice. Qual Prim Care 2016;24:289-92.

22. von Wagner $\mathrm{C}$, Stoffel S, Freeman M, et al. Attitudes towards faecal immunochemical testing in patients at increased risk of colorectal cancer: an online survey of GPs in England. Br J Gen Pract 2018;68:e757-e764.

23. Kidney E, Greenfield S, Berkman L, et al. Cancer suspicion in general practice, urgent referral, and time to diagnosis: a populationbased GP survey nested within a feasibility study using information technology to flag-up patients with symptoms of colorectal cancer. BJGP Open 2017;1:BJGP-2016-0725.

24. Nicholson BD, Oke JL, Rose PW, et al. Variation in direct access to tests to investigate cancer: A survey of english general practitioners. PLoS One 2016;11:e0159725.

25. Green T, Atkin K, Macleod U. Cancer detection in primary care: insights from general practitioners. Br J Cancer 2015;112 Suppl 1(Suppl 1):S41-S49.

26. National Institute for Health and Care Excellence. Adoption support resource - Insights from the NHS. 2018 https://www.nice.org.uk/ guidance/dg30/resources/adoption-support-for-quantitative-faecalimmunochemical-tests-to-guide-referral-for-colorectal-cancerin-primary-care-insights-from-the-nhs-pdf-220288617251125 (Accessed 30 Oct 2018). 